STOCHASTIC MODELING AND CONTROL
BANACH CENTER PUBLICATIONS, VOLUME 122
INSTITUTE OF MATHEMATICS
POLISH ACADEMY OF SCIENCES
WARSZAWA 2020

\title{
ON THE MINIMAX THEOREM FOR THE SPACE OF PROBABILITY MEASURES ON METRIC SPACES
}

\author{
DANIEL HERNÁNDEZ-HERNÁNDEZ \\ Department of Probability and Statistics, Research Center for Mathematics \\ Apartado postal 402, Guanajuato, Gto. 36000, Mexico \\ ORCID:0000-0002-3948-9179_E-mail:dher@cimat.mx
}

JOSÉ VILLA-MORALES

Departamento de Matemáticas y Física, Universidad Autónoma de Aguascalientes

Aguascalientes, Ags., Mexico

ORCID:0000-0002-2263-136X_E-mail: jvilla@correo.uaa.mx

\begin{abstract}
Using Prohorov's Theorem we give a proof of the Minimax Theorem in the context of probability measures defined on separable metric spaces. We also introduce the concept of pseudo-characteristic function and use it to give necessary and sufficient conditions of relative compactness in the space of probability measures.
\end{abstract}

1. Introduction. This paper concerns the zero-sum static game represented by strategies of players with opposite objectives. Motivated by well known results (named in the literature as Minimax theorem) in static games ensuring the existence of value of the game, when mixed strategies are allowed for both players and the corresponding set of actions $E$ and $F$ for both players are compact, we extend those results to the case when the payoff function $f$ is measurable and the minimizer action space is not necessarily compact.

The interest in this extension comes from different fields, where the compactness assumption and the continuity of the payoff function are not satisfied; in fact are not natural hypotheses in view of the nature of the problem at hand. See, for instance,

2010 Mathematics Subject Classification: Primary 49K35; Secondary 91A25.

Key words and phrases: Minimax theorem, mixed strategies, zero-sum static games, pseudocharacteristic function.

The paper is in final form and no version of it will be published elsewhere. 
[21], [10, [9], or 20] for the study of the Minimax theorem for fuzzy metric spaces. Also, in the study of deterministic differential games with mixed strategies, the Minimax theorem appears at a local level, meaning that when the Hamiltonians are analyzed for the upper and lower values, an interesting question to be answered is if it is possible to interchange the inf and sup in their definition, as in 11. Consequently, standard statements of the Minimax theorem [2], 5] cannot be applied to establish the existence of equilibrium strategies or a saddle point.

The pioneering work of von Neumann in 1928 established the existence of a saddle point for games with finite action spaces with the discrete metric. This classical result has been extended in different directions; here we mention a few of them, since the existence literature related with this theory is abundant. For instance, concerning the topology/geometry of the action spaces for the players, in [14, [16], [12] the authors consider nonconvex and non-Hausdorff spaces. Also, for Hilbert and Lindelöf spaces the conclusion of the Minimax theorem has been analyzed in [4] and 24].

Concerning the payoff function, some examples of structure that has been studied are the following. In [10] and 25] the case with semi-continuous functions was considered, whereas in [23], the authors consider linear operators. Also, when the payoff function is generalized as a set-valued map, the minimax problem was analyzed in [18] or [15].

In the present paper we study the Minimax theorem when the action space is a separable metric space, and the proportion of times that each player choose a particular pure strategy has certain probability distribution, defined on the Borel sigma algebra. These are called mixed strategies. When the action space of the minimizer player is not compact we present some interesting conclusions. In order to study this case we introduce the concept of pseudo-characteristic functions on proper metric spaces and we use this concept to give a criterion of tightness, which is closely related to the compactness.

The organization of the paper is as follows. In the next section some preliminary results are stated for static games, describing also in a precise way the main objective of this work. In Section 3 we present the main conclusions, providing a proof of the Minimax Theorem in the context of probability measures on separable metric spaces. Finally, in Section 4 tightness is characterized in terms of pseudo-characteristic functions and an example is presented, where such characterization is used.

2. Preliminaries on static games. Given metric spaces $(E, d)$ and $(F, m)$, let us consider a static game with a bounded measurable payoff function $f(x, y)$, with $x \in E$ and $y \in F$. The upper and lower values are defined as

$$
\hat{V}^{+}=\inf _{x \in E} \sup _{y \in F} f(x, y) \quad \text { and } \quad \hat{V}^{-}=\sup _{x \in E} \inf _{y \in F} f(x, y) .
$$

Somehow the advantage of information for each player is reflected in the order of the sup or inf. For instance, in the definition of $\hat{V}^{+}$the maximizer player has an information advantage, since $y$ can be chosen as a function of $x$. This can be made precise using decision rules $\alpha: E \rightarrow F$ and writing $\hat{V}^{+}=\inf _{\alpha} \sup _{x \in E} f(x, \alpha(x))$. A similar argument can be used for the lower value $\hat{V}^{-}=\sup _{\beta} \inf _{y \in F} f(\beta(y), y)$, where the infimum is taken over the set of decision rules $\beta: F \rightarrow E$. In fact, under some topological conditions on the 
metric spaces, it can be proved that optimal strategies and decision rules can be chosen for each player.

The inequality $\hat{V}^{-} \leq \hat{V}^{+}$is valid in general. In the case when the upper and lower values are equal we say that neither player has an advantage and the game has a value, the value of the game is the common amount. Under suitable topological conditions, there exist saddle points, points where the value of the game is reached, and they can be found by solving a sequential optimization problem, for $\hat{V}^{-}$, for instance, we proceed as follows: first we define $\beta^{*}(y)=\arg \min _{x \in E} f(x, y)$, and then

$$
y^{*}=\underset{y}{\arg \max } \inf _{x} f(x, y)=\underset{y}{\arg \max } f\left(\beta^{*}(y), y\right) .
$$

When the strict inequality $\hat{V}^{-}<\hat{V}^{+}$holds, the "pure" strategies can be defined through mixed strategies in order to get a value of the game, meaning that a randomization procedure can be implemented where the players select probability measures in the set of actions, from which the players select their decisions from random sampling.

More precisely, if we denote by $\tau_{E}$ the collection of all open subsets of the metric space $(E, \rho)$ and by $\mathcal{B}_{E}=\sigma\left(\tau_{E}\right)$ the Borel $\sigma$-algebra on $E$, the family of probability measures on $E$ is denoted by $\mathcal{P}(E)$. The same notation is used when $E$ is substituted by the other metric space $F$. The spaces $\mathcal{P}(E)$ and $\mathcal{P}(F)$ are endowed with the weak topology; it is well known that with this topology these spaces are metrizable. Indeed, one metric is the Prohorov's metric in $\mathcal{P}(E)$ which is defined as

$$
\rho_{\mathcal{P}(E)}(P, Q)=\inf \left\{\varepsilon>0: P(A) \leq Q\left(A_{\varepsilon}\right)+\varepsilon \text { for all } E \backslash A \in \tau_{E}\right\},
$$

where $A_{\varepsilon}=\{x \in E: \varrho(x, A)<\varepsilon\}$ with $\varrho(x, A)=\inf _{y \in A} \varrho(x, y)$.

For static games, when the players are allowed to consider as strategies probability measures in $\mathcal{P}(E)$ and $\mathcal{P}(F)$, we say that they are using mixed strategies. This type of strategies can be described as if the game were repeated indefinitely and the average results in the expression

$$
J(P, Q):=\int_{E} \int_{F} f(x, y) Q(d y) P(d x),
$$

which represents the corresponding payoff associated to the strategy $P \in \mathcal{P}(E)$ and $Q \in \mathcal{P}(F)$ for the minimizer and maximizer players, respectively. Then, the upper and lower values of the game, analogous to (1), are defined as

$$
\begin{aligned}
V^{+} & =\inf _{P \in \mathcal{P}(E)} \sup _{Q \in \mathcal{P}(F)} J(P, Q), \\
V^{-} & =\sup _{Q \in \mathcal{P}(F)} \inf _{P \in \mathcal{P}(E)} J(P, Q) .
\end{aligned}
$$

As it was defined above for pure strategies, when the values (4) and (5) are equal the common value is called the value of the game, and if this common value is reached at a point $\left(P_{0}, Q_{0}\right)$ we say that it is a saddle point. Intuitively it means that if any of the players deviates from their optimal strategy, $\left(P_{0}, Q_{0}\right)$, they can only give an advantage to the opposite player.

Since the function $(P, Q) \mapsto J(P, Q)$ is bilinear, it is well known that if $f$ is continuous and the action spaces $E$ and $F$ are compact, there exists a value of the game, and there 
is a pair of measures $\left(P^{*}, Q^{*}\right)$ such that, for every pair of probability measures $(P, Q)$,

$$
J\left(P^{*}, Q\right) \leq J\left(P^{*}, Q^{*}\right) \leq J\left(P, Q^{*}\right) .
$$

That is, the inf and sup can be interchanged in expressions (4)-(5); see, for instance, [2] p. 218]. Recall that under this compactness assumption the spaces $\mathcal{P}(E)$ and $\mathcal{P}(F)$ are also compact under the weak topology. Hence, in this particular case, $V^{+}=V^{-}$.

Roughly speaking, when this interchange of supremum and infimum is possible we say that the Minimax theorem holds, as it will be recalled in the next section. In this note we are interested in the extension of (6) to the case when the payoff function $f$ is measurable and l.s.c. in the minimizer action variable. These are nonstandard conditions from a technical viewpoint.

3. The Minimax theorem. In order to present the main results of this note, we first introduce some notation and classical statements of the Minimax theorem. Let $X$ and $Y$ be separated (Hausdorff) locally convex topological vector spaces and $L: X \times Y \rightarrow \mathbb{R}$ a real valued function defined in the product space.

Definition 3.1. We say that $L: X \times Y \rightarrow \mathbb{R}$ is convex-concave if $L(\cdot, y)$ is convex for all $y \in Y$ and $L(x, \cdot)$ is concave for all $x \in X$. Analogously, we say that $L$ is l.s.c.-u.s.c. if $L(\cdot, y)$ is lower semi-continuous for all $y \in Y$ and $L(x, \cdot)$ is upper semi-continuous for all $x \in X$.

Next version of the Minimax theorem is one of the classical versions of that result. It states the existence of value for the static game under suitable technical conditions; its proof can be found in [26] or, for a modern treatment, the interested reader can also find it in [19] or 3]. This result also represents an extension of the fundamental result of von Neumann proved in 1928 in the context of the simplex sets.

THEOREM 3.2. Let $E$ and $F$ be nonempty convex subsets of $X$ and $Y$, respectively, with $E$ compact. Let $L: E \times F \rightarrow \mathbb{R}$ be convex-concave and $L(\cdot, y)$ l.s.c. for each $y \in F$. Then

$$
\sup _{y \in F} \inf _{x \in E} L(x, y)=\inf _{x \in E} \sup _{y \in F} L(x, y),
$$

and there exists $x_{0} \in E$ such that

$$
\sup _{y \in F} L\left(x_{0}, y\right)=\inf _{x \in E} \sup _{y \in F} L(x, y) .
$$

As an immediate consequence we have the existence of saddle points; see Theorem 11.5 in [13] for its proof.

Corollary 3.3. Let $E$ and $F$ be nonempty convex and compact subsets of $X$ and $Y$, respectively. Let $L: E \times F \rightarrow \mathbb{R}$ be convex-concave and l.s.c.-u.s.c.. Then, there exists a point $\left(x_{0}, y_{0}\right) \in E \times F$ such that

$$
L\left(x_{0}, y\right) \leq L\left(x_{0}, y_{0}\right) \leq L\left(x, y_{0}\right), \quad \text { for all } x \in X, y \in Y,
$$

and, in particular,

$$
\min _{x \in X} \sup _{y \in Y} L(x, y)=L\left(x_{0}, y_{0}\right)=\max _{x \in X} \inf _{y \in Y} L(x, y)
$$


Our attempt now is to apply the above results to prove the existence of value of the game defined in (4)-(5), and a saddle point, satisfying (6), within the context of mixed strategies and the payoff function $J$, as it was defined in (3).

The main result of this note is the following. Its proof requires the concept of tightness of a family of probability measures, which we recall for completeness. Given a metric space $S$, a family $\left\{\mu_{\alpha}\right\} \subset P(S)$ is tight if for each $\varepsilon>0$ there exists a compact set $K \subset S$ such that

$$
\inf _{\alpha} \mu_{\alpha}(K) \geq 1-\varepsilon .
$$

THEOREM 3.4. Let $E, F$ be separable metric spaces, with $F$ compact. Let $f: E \times F \rightarrow \mathbb{R}$ be a bounded measurable function such that $f(\cdot, y)$ is l.s.c. for each $y \in F$. Then,

$$
\sup _{\nu \in \mathcal{P}(F)} \inf _{\mu \in \mathcal{P}(E)} \int_{E} \int_{F} f(x, y) \nu(d y) \mu(d x)=\inf _{\mu \in \mathcal{P}(E)} \sup _{\nu \in \mathcal{P}(F)} \int_{E} \int_{F} f(x, y) \nu(d y) \mu(d x) .
$$

Proof. We proceed to verify the hypotheses of Theorem 3.2 in order to apply that result. It is clear that $\mathcal{P}(E)$ and $\mathcal{P}(F)$ are convex sets (moreover, if instead of measures we consider signed measures then they are linear spaces over the real numbers). Since $E$ is compact, $\mathcal{P}(E)$ is also compact; see [17, Theorem 6.4]. Let us define the function $L: \mathcal{P}(E) \times \mathcal{P}(F) \rightarrow \mathbb{R}$ by

$$
L(\mu, \nu)=\int_{E} \int_{F} f(x, y) \nu(d y) \mu(d x),
$$

so, we are done if we prove that $L(\cdot, \nu)$ is l.s.c. for each $\nu \in \mathcal{P}(F)$.

Let $\nu \in \mathcal{P}(F)$ be a fix probability measure. For each $r \in \mathbb{R}$ we need to verify that the set (called section) defined by

$$
A_{r}(\nu)=\left\{\mu \in \mathcal{P}(E): \int_{E} \int_{F} f(x, y) \nu(d y) \mu(d x) \leq r\right\}
$$

is closed in $\mathcal{P}(E)$. Let $\left(\mu_{n}\right)$ be a sequence in the section $A_{r}(\nu)$, such that

$$
\lim _{n \rightarrow \infty} \rho_{\mathcal{P}(E)}\left(\mu_{n}, \mu\right)=0 ;
$$

see (2). This implies that the set $\left\{\mu_{n}: n \in \mathbb{N}\right\} \cup\{\mu\}$ is compact, and then $\left\{\mu_{n}: n \in \mathbb{N}\right\}$ is relatively compact, concluding that $\left\{\mu_{n}: n \in \mathbb{N}\right\}$ is tight by Prohorov's theorem; see, for instance, [8, Theorem 3.2.2]). Let us check that $\left\{\mu_{n} \times \nu: n \in \mathbb{N}\right\}$ is also tight. Since $\{\nu\}$ is compact it is also tight by Prohorov's theorem. Therefore, for each $\varepsilon>0$ there are compacts sets $K_{1} \subset E, K_{2} \subset F$ such that

$$
\mu_{n}\left(K_{1}\right) \geq|1-\varepsilon|^{1 / 2}, \text { for all } n \in \mathbb{N},
$$

and $\nu\left(K_{2}\right) \geq|1-\varepsilon|^{1 / 2}$. For the compact set $K_{1} \times K_{2} \subset E \times F$ we have

$$
\left(\mu_{n} \times \nu\right)\left(K_{1} \times K_{2}\right)=\mu_{n}\left(K_{1}\right) \nu\left(K_{2}\right) \geq 1-\varepsilon, \text { for all } n \in \mathbb{N},
$$

and Prohorov's theorem implies that $\left\{\mu_{n} \times \nu: n \in \mathbb{N}\right\}$ is relatively compact.

Now, we proceed to verify that every convergent subsequence of $\left\{\mu_{n} \times \nu\right\}$ converges to $\mu \times \nu$. Let $\left\{\mu_{n_{j}} \times \nu\right\}$ be a subsequence of $\left\{\mu_{n} \times \nu\right\}$ converging to $m \in \mathcal{P}(E \times F)$. Let $A \subset E, B \subset F$ be closed sets and for each $k \in \mathbb{N}$ define $g_{k}: E \rightarrow \mathbb{R}, h_{k}: F \rightarrow \mathbb{R}$ as

$$
g_{k}(x)=\max \{0,1-k d(x, A)\}, \quad h_{k}(y)=\max \{0,1-k d(y, B)\} .
$$


The functions $(x, y) \mapsto g_{k}(x) h_{k}(y)$, defined on $E \times F$, are bounded and continuous and $\lim _{k \rightarrow \infty} g_{k} h_{k}=1_{A} 1_{B}$. The dominated convergence theorem yields (see Theorem 3.3.1 in [8]):

$$
\begin{aligned}
& \int_{E \times F} 1_{A}(x) 1_{B}(y) m(d x, d y)=\lim _{k \rightarrow \infty} \int_{E \times F} g_{k}(x) h_{k}(y) m(d x, d y) \\
& =\lim _{k \rightarrow \infty} \lim _{j \rightarrow \infty} \int_{E \times F} g_{k}(x) h_{k}(y)\left(\mu_{n_{j}} \times \nu\right)(d x, d y) \\
& =\lim _{k \rightarrow \infty} \lim _{j \rightarrow \infty} \int_{E} g_{k}(x) \mu_{n_{j}}(d x) \int_{F} h_{k}(y) \nu(d y) \\
& =\lim _{k \rightarrow \infty} \int_{E} g_{k}(x) \mu(d x) \int_{F} h_{k}(y) \nu(d y)=\int_{E} 1_{A}(x) \mu(d x) \int_{F} 1_{B}(y) \nu(d y),
\end{aligned}
$$

therefore

$$
m(A \times B)=(\mu \times \nu)(A \times B), \text { for all } A \subset E, B \subset F, \text { closed. }
$$

Since $E$ and $F$ are separable we can apply Lindelöf theorem to get $\tau_{E \times F} \subset \sigma\left(\tau_{E}^{c} \times \tau_{F}^{c}\right)$, where $\tau_{E}^{c} \times \tau_{F}^{c}=\left\{A \times B: E \backslash A \in \tau_{E}, F \backslash B \in \tau_{F}\right\}$, and therefore $\sigma\left(\tau_{E \times F}\right) \subset \sigma\left(\tau_{E}^{c} \times \tau_{F}^{c}\right) \subset$ $\sigma\left(\tau_{E \times F}\right)$. Since $\tau_{E}^{c} \times \tau_{F}^{c}$ is a $\pi$-system we conclude that $m=\mu \times \nu$, by [6. Corollary 1.6.3].

If $\left(\mu_{n} \times \nu\right)$ does not converge to $\mu \times \nu$, then there exist $\varepsilon_{0}>0$ and a subsequence $\left(\mu_{n_{j}} \times \nu\right)$ such that

$$
\rho_{\mathcal{P}(E \times F)}\left(\mu_{n_{j}} \times \nu, \mu \times \nu\right) \geq \varepsilon_{0}, \text { for all } j \in \mathbb{N} .
$$

Since $\left\{\mu_{n_{j}} \times \nu: j \in \mathbb{N}\right\}$ is relatively compact, there exists a subsequence $\left(\mu_{n_{j_{k}}} \times \nu\right)$ that converge to $\mu \times \nu$. This is a contradiction to (8).

The last step in the proof consists in verifying that $\mu \in A_{r}(\nu)$. Let

$$
\|f\|=\sup \{|f(x, y)|: x \in E, y \in F\},
$$

and notice that by Fatou's lemma

$$
\begin{aligned}
& \int_{F} \int_{0}^{\|f\|} \liminf _{n \rightarrow \infty}\left[1-\mu_{n}(\{x \in E: f(x, y) \leq t\})\right] d t \nu(d y) \\
& \leq \liminf _{n \rightarrow \infty} \int_{F} \int_{0}^{\|f\|}\left[1-\mu_{n}(\{x \in E: f(x, y) \leq t\})\right] d t \nu(d x) .
\end{aligned}
$$

Since $f$ is l.s.c. the set $\{x \in E: f(x, y) \leq t\}$ is closed, for each $t \in \mathbb{R}$ and $y \in F$. Using Fubini's theorem, Theorem 3.3.1 in [8] and $(9)$ we get

$$
\begin{aligned}
\|f\|-r & \leq\|f\|-\liminf _{n \rightarrow \infty} \int_{E \times F} f(x, y)\left(\mu_{n} \times \nu\right)(d x, d y) \\
& =\limsup _{n \rightarrow \infty}\left(\|f\|-\int_{E \times F} f(x, y)\left(\mu_{n} \times \nu\right)(d x, d y)\right) \\
& =\limsup _{n \rightarrow \infty} \int_{F}\left(\|f\|-\int_{E} f(x, y) \mu_{n}(d x)\right) \nu(d y) \\
& =\limsup _{n \rightarrow \infty} \int_{F} \int_{0}^{\|f\|} \mu_{n}(\{x \in E: f(x, y) \leq t\}) d t \nu(d x) \\
& \leq \int_{F} \int_{0}^{\|f\|} \limsup _{n \rightarrow \infty} \mu_{n}(\{x \in E: f(x, y) \leq t\}) d t \nu(d x)
\end{aligned}
$$




$$
\begin{aligned}
& \leq \int_{F} \int_{0}^{\|f\|} \mu(\{x \in E: f(x, y) \leq t\}) d t \nu(d x) \\
& =\int_{F}\left(\|f\|-\int_{E} f(x, y) \mu(d x)\right) \nu(d y)=\|f\|-\int_{E \times F} f(x, y)(\mu \times \nu)(d x, d y) .
\end{aligned}
$$

This shows that $\mu \in A_{r}(\nu)$ and the result follows directly from Theorem 3.2

From this point, the previous result together with Corollary 3.3 yield as a straightforward consequence the existence of saddle points.

Corollary 3.5. Let $E$ and $F$ be compact metric spaces. Let $f: E \times F \rightarrow \mathbb{R}$ be a bounded measurable function that is l.s.c.-u.s.c. Then, there exists a point $\left(\mu_{0}, v_{0}\right) \in \mathcal{P}(E) \times \mathcal{P}(F)$ such that

$$
\begin{aligned}
\sup _{\nu \in \mathcal{P}(F)} \inf _{\mu \in \mathcal{P}(E)} \int_{E} \int_{F} f(x, y) \nu(d y) \mu(d x) & =\int_{E} \int_{F} f(x, y) \nu_{0}(d y) \mu_{0}(d x) \\
& =\inf _{\mu \in \mathcal{P}(E)} \sup _{\nu \in \mathcal{P}(F)} \int_{E} \int_{F} f(x, y) \nu(d y) \mu(d x) .
\end{aligned}
$$

4. Pseudo-characteristic functions on proper metric spaces and tightness. A locally compact metric space $(S, \varrho)$ is said to be proper (see [22, p. 204]) if the closed balls $\left\{x: \varrho\left(x, x_{0}\right) \leq r\right\}$ are compact for some $x_{0}$ and all $r \in(0, \infty)$. It is easy to show that tightness condition (7) is equivalent to

$$
\lim _{r \rightarrow \infty} \sup _{\alpha} \mu_{\alpha}\left\{x: \varrho\left(x, x_{0}\right)>r\right\}=0 .
$$

The pseudo-characteristic function of a measure $\mu \in P(S)$ is defined as

$$
\hat{\mu}(t)=\int_{S} e^{i t d\left(z, x_{0}\right)} \mu(d z), \quad t \in \mathbb{R} ;
$$

observe that $|\hat{\mu}(t)| \leq 1=\hat{\mu}(0)$. As it has been seen in the proof of Theorem 3.4 tightness of a family of probability measures plays a crucial role. The following result is particularly useful when we do not have compactness in the action space of the players.

THEOREM 4.1. A family $\left\{\mu_{\alpha}\right\} \subset P(S)$ is tight if and only if $\left\{\hat{\mu}_{\alpha}\right\}$ is equicontinuous at 0 . Proof. Suppose that $\left\{\mu_{\alpha}\right\} \subset P(S)$ is tight. Given $\varepsilon>0$ there exists $r_{0}>0$ such that

$$
\sup _{\alpha} \mu_{\alpha}\left\{x: \varrho\left(x, x_{0}\right)>r\right\}<\frac{\varepsilon}{2}, \text { for all } r \geq r_{0} .
$$

Therefore, if $|t|<\min \{2, \varepsilon\} / 2 r_{0}$, then

$$
\begin{aligned}
\left|\hat{\mu}_{\alpha}(t)-1\right| & \leq \int_{S}\left|1-e^{i t d\left(z, x_{0}\right)}\right| \mu_{\alpha}(d z) \leq \int_{S} \min \left\{1, t d\left(z, x_{0}\right)\right\} \mu_{\alpha}(d z) \\
& \leq \int_{\left\{z: d\left(z, x_{0}\right)>r_{0}\right\}} \mu_{\alpha}(d z)+\int_{\left\{z: d\left(z, x_{0}\right) \leq r_{0}\right\}} t d\left(z, x_{0}\right) \mu_{\alpha}(d z) \\
& \leq \mu_{\alpha}\left(\left\{z: d\left(z, x_{0}\right)>r_{0}\right\}\right)+t r_{0}<\varepsilon, \text { for all } \alpha .
\end{aligned}
$$


In order to prove the reciprocal implication, take $r>0$ and observe that by Fubini's theorem, for each $\alpha$ we have

$$
\begin{aligned}
& \int_{-2 / r}^{2 / r}\left(1-\hat{\mu}_{\alpha}(t)\right) d t=\int_{-2 / r}^{2 / r} \int_{S}\left(1-e^{i t d\left(z, x_{0}\right)}\right) \mu_{\alpha}(d z) d t \\
& \quad=\int_{S}\left[r-\int_{-2 / r}^{2 / r} e^{i t d\left(z, x_{0}\right)} d t\right] \mu_{\alpha}(d z)=r \int_{S}\left[1-\frac{2}{r d\left(z, x_{0}\right)} \sin \left(\frac{r d\left(z, x_{0}\right)}{2}\right)\right] \mu_{\alpha}(d z) .
\end{aligned}
$$

Since $2 \sin (x) \leq x$, for $x \geq 2$, it follows that

$$
\int_{-2 / r}^{2 / r}\left(1-\hat{\mu}_{\alpha}(t)\right) d t \geq r \int_{\left\{z: d\left(z, x_{0}\right) \geq r\right\}} \frac{1}{2} \mu_{\alpha}(d z)=\frac{2}{r} \mu_{\alpha}\left(\left\{z: \varrho\left(z, x_{0}\right) \geq r\right\}\right) .
$$

Now, given $\varepsilon>0$, there exists $\delta>0$ for which

$$
1-\hat{\mu}_{\alpha}(t)<\frac{\varepsilon}{2}, \quad \text { for all }|t|<\delta \text { and } \alpha .
$$

In this way, for $r>4 / \delta$,

$$
\mu_{\alpha}\left\{x: \varrho\left(x, x_{0}\right)>r\right\} \leq \int_{-1}^{1}\left(1-\hat{\mu}_{\alpha}\left(\frac{2}{r} t\right)\right) d t \leq \varepsilon, \text { for all } \alpha,
$$

and therefore $\lim _{r \rightarrow \infty} \sup _{\alpha} \mu_{\alpha}\left\{x: \varrho\left(x, x_{0}\right)>r\right\}=0$.

Now we present an example illustrating the difficulties to get the conclusions of the Minimax theorem when the action spaces are not compact, as a complement of the previous results.

ExAmPLE. Let us take $E=\{1,2, \ldots\}$ with the usual distance $d$ on $\mathbb{R}$, and put $x_{0}=1$, in such a way that $(E, d)$ is a proper metric space. On the other hand, let $F=\{1,2, \ldots\}$, with the discrete metric $m$, be the set of actions for the maximizer player. Suppose that the minimizer player chooses a strategy $k \in E$ with frequency $\mu_{\alpha}(\{k\})=(1-\alpha)^{k-1} \alpha$, $\alpha \in[1 / 2,1]$. In this case, by Theorem 4.1. the closure of $\left\{\mu_{\alpha}\right\}_{\alpha \in[1 / 2,1]}$ is compact, since

$$
\hat{\mu}_{\alpha}(t)=\int_{E} e^{i t|z-1|} \mu_{\alpha}(d z)=\sum_{k=1}^{\infty} e^{i t(k-1)}(1-\alpha)^{k-1} \alpha=\frac{\alpha}{1-e^{i t}(1-\alpha)}
$$

is equicontinuous at 0 . Indeed, this follows from next calculations

$$
\left|\frac{\alpha}{1-e^{i t}(1-\alpha)}-1\right|=\frac{(1-\alpha)\left|1-e^{i t}\right|}{\left|1-e^{i t}(1-\alpha)\right|} \leq \frac{(1-\alpha)\left|1-e^{i t}\right|}{1-\left|e^{i t}(1-\alpha)\right|} \leq\left|1-e^{i t}\right|, \text { for all } \frac{1}{2} \leq \alpha \leq 1 \text {. }
$$

Let $f: E \times F \rightarrow \mathbb{R}$ be a bounded function, representing the payoff function for the players. We denote by $C(E, F)$ the set of continuous mappings from $E$ to $F$, they shall represent decision rules for the maximizer player. In order to use Theorem 8.4 in [3] let us observe that for each $g \in C(E, F)$, the mapping $T: \mathcal{P}(E) \rightarrow \mathcal{P}(F)$ defined as

$$
T(\mu)(A)=\mu^{g}(A)=\mu\left(g^{-1}(A)\right), \quad A \in \mathcal{B}(F),
$$


is continuous. Therefore, the change of variable theorem (see Theorem 12.46 in [1]) yields

$$
\begin{aligned}
& \sup _{g \in C(E, F)} \inf _{\alpha \in[1 / 2,1]} \int_{E} \int_{F} f(x, g(y)) \mu_{\alpha}(d y) \mu_{\alpha}(d x) \\
& =\sup _{g \in C(E, F)} \inf _{\alpha \in[1 / 2,1]} \int_{E} \int_{F} f(x, y) \mu_{\alpha}^{g}(d y) \mu_{\alpha}(d x) \\
& =\inf _{\alpha \in[1 / 2,1]} \sup _{v \in \mathcal{P}(F)} \int_{E} \int_{F} f(x, y) v(d y) \mu_{\alpha}(d x) \\
& =\inf _{\alpha \in[1 / 2,1]} \sup _{g \in C(E, F)} \int_{E} \int_{F} f(x, y) \mu_{\alpha}^{g}(d y) \mu_{\alpha}(d x) \\
& =\inf _{\alpha \in[1 / 2,1]} \sup _{g \in C(E, F)} \int_{E} \int_{F} f(x, g(y)) \mu_{\alpha}(d y) \mu_{\alpha}(d x) .
\end{aligned}
$$

The result intuitively gives a representation of the upper value of the game, when the maximizer player is forced to use only continuous decision rules and the minimizer player takes mixed strategies within the set $\left\{\mu_{\alpha}: \alpha \in\left[\frac{1}{2}, 1\right]\right\}$.

In particular, when the continuous decision rule for the maximizer player is the identity, $g(k)=k$, for all $k$, with payoff function $f(x, y)=e^{-y} y^{x} / x$ !, we get a lower bound for the upper value

$$
\begin{aligned}
& \inf _{\alpha \in[1 / 2,1]} \alpha^{2} \sum_{r=1}^{\infty} \sum_{s=1}^{\infty} \frac{e^{-s} s^{r}}{r !}(1-\alpha)^{r-1}(1-\alpha)^{s-1} \\
& =\inf _{\alpha \in[1 / 2,1]} \alpha^{2} \sum_{s=1}^{\infty} e^{-s}(1-\alpha)^{s-1} \sum_{r=1}^{\infty}(1-\alpha)^{r-1} \frac{s^{r}}{r !} \\
& =\inf _{\alpha \in[1 / 2,1]} \alpha^{2} \sum_{s=1}^{\infty} e^{-s}(1-\alpha)^{s-1}\left[\frac{1}{\alpha-1}-\frac{e^{-s(\alpha-1)}}{\alpha-1}\right] \\
& =\inf _{\alpha \in[1 / 2,1]} \frac{e^{-\alpha}-e^{-1}}{1-\alpha} \cdot \frac{1}{\left(\alpha e^{-1}-e^{-1}+1\right)\left(\alpha e^{-\alpha}-e^{-\alpha}+1\right)}=\frac{1}{e} .
\end{aligned}
$$

Under these conditions, the maximum loss in which the minimizer player might incur, in average, is bigger than 0.367 .

Acknowledgments. Research of D. Hernández-Hernández was supported by Conacyt Grant 254166 and J. Villa-Morales received financial support from Universidad Autónoma de Aguascalientes through grant PIM20-1.

\section{References}

[1] C. D. Aliprantis, K. C. Border, Infinite Dimensional Analysis: A Hitchhiker's Guide, Springer, Berlin, 1999.

[2] J.-P. Aubin, Mathematical Methods of Game and Economic Theory, Stud. Math. Appl. 7, North Holland, Amsterdam, 1979.

[3] J.-P. Aubin, Optima and Equilibria, an Introduction to Nonlinear Analysis, Grad. Texts in Math. 140, Springer, Berlin, 1998. 
[4] E. M. Bednarczuk, M. Syga, On minimax theorems for lower semicontinuous functions in Hilbert spaces, J. Convex Anal. 25 (2018), 389-402.

[5] J. Borwein, A very complicated proof of the minimax theorem, Minimax Theory Appl. 1 (2016), 21-27.

[6] D. L. Cohn, Measure Theory, Birkhäuser Adv. Texts Basler Lehrbücher, Springer, New York, 2013.

[7] R. M. Dudley, Real Analysis and Probability, Cambridge Stud. Adv. Math. 74, Cambridge Univ. Press, Cambridge, 2002.

[8] S. N. Ethier, T. G. Kurtz, Markov Processes, Characterization and Convergence, Wiley Ser. Probab. Math. Statist. Wiley, New York, 1986.

[9] E. A. Feinberg, P. O. Kasyanov, M. Z. Zgurovsky, Continuity of equilibria for two-person zero-sum games with noncompact action sets and unbounded payoffs, Ann. Oper. Res. (October 2017).

[10] E. A. Feinberg, P. O. Kasyanov, M. Z. Zgurovsky, An example showing that A-lower semicontinuity is essential for minimax continuity theorems, Oper. Res. Lett. 46 (2018), 385-388.

[11] W. Fleming, D. Hernández-Hernández, Mixed strategies for deterministic differential games, Commun. Stoch. Anal. 11 (2017), 137-156.

[12] J. Goubault-Larrecq, A non-Hausdorff minimax theorem, Minimax Theory Appl. 3 (2018), $73-80$.

[13] O. Güler, Foundations of Optimization, Grad. Texts in Math. 258, Springer, New York, 2010.

[14] E. Hashemi, R. Saadati, EVP, Minimax theorems and existence of nonconvex equilibria in complete G-metric spaces, J. Comput. Anal. Appl. 25 (2018), 441-451.

[15] I. Kuwano, Some minimax theorems of set-valued maps and their applications, Nonlinear Anal. 109 (2014), 85-102.

[16] B. S. Lee, Generalized minimax theorems on nonconvex domains, Appl. Math. E-Notes 15 (2015), 46-53.

[17] K. R. Parthasarathy, Probability Measures on Metric Spaces, Amer. Math. Soc., Chelsea Publishing, Providence, RI, 1967.

[18] M. Patriche, Minimax theorems for set-valued maps without continuity assumptions, Optimization 65 (2016), 957-976.

[19] J. Ponstein, Approaches to the Theory of Optimization, Cambridge Tracts in Math. 77, Cambridge Univ. Press, Cambridge, 1980.

[20] M. H. M. Rashid, Minimax theorems in fuzzy metric spaces, Comput. Appl. Math. 37 (2018), 1703-1720.

[21] B. Ricceri, On a minimax theorem: an improvement, a new proof and an overview of its applications, Minimax Theory Appl. 2 (2017), 99-152.

[22] H. L. Royden, Real Analysis, Macmillan, New York, 1988.

[23] J. Saint Raymond, A new minimax theorem for linear operators, Minimax Theory Appl. 3 (2018), 131-160.

[24] C. Sun, A minimax theorem for Lindelöf sets, J. Optim. Theory Appl. 179 (2018), 127-136.

[25] G. Tian, Full characterizations of minimax inequality, fixed point theorem, saddle point theorem, and KKM principle in arbitrary topological spaces, J. Fixed Point Theory Appl. 19 (2017), 1679-1693.

[26] H. Tuy, On a general minimax theorem, Soviet Math. Dokl. 15 (1974), 1689-1693.

[27] Q. B. Zhang, General two-function topological minimax theorems, Ann. Oper. Res. 217 (2014), 591-598. 